\title{
Association of Arterial Pressure Volume Index With the Presence of Significantly Stenosed Coronary Vessels
}

\author{
Takashi Ueda ${ }^{a}$, Shin-ichiro Miura ${ }^{a}$, b, d, Yasunori Suematsu ${ }^{a}$, Yuhei Shiga ${ }^{a}$, Takashi Kuwano, \\ Makoto Sugihara $^{\mathrm{a}}$, Amane Ike ${ }^{\mathrm{a}}$, Atsushi Iwata ${ }^{\mathrm{a}}$, Hiroaki Nishikawa ${ }^{\mathrm{a}}$, Kanta Fujimi ${ }^{\mathrm{a}}$, , \\ Keijiro Saku ${ }^{\mathrm{a}, \mathrm{b}}$
}

\begin{abstract}
Background: A blood pressure (BP) monitoring system (PASESA ${ }^{\circledR}$ ) can be used to easily analyze the characteristics of central and peripheral arteries during the measurement of brachial BP.

Methods: We enrolled 108 consecutive patients $(\mathrm{M} / \mathrm{F}=86 / 22$, age $70 \pm 10$ years) who underwent coronary angiography (CAG) due to suspected coronary artery disease (CAD) in whom we could measure various parameters using PASESA ${ }^{\circledR}$ in addition to brachial-ankle pulse wave velocity (baPWV). The patients were divided into two groups: patients who did not have significantly stenosed coronary vessel disease ( $n=33$, non-SVD group) and those who had at least one significantly stenosed coronary vessel ( $n=75$, SVD group). The characteristics of central and peripheral arteries (arterial velocity pulse index (AVI) and arterial pressure volume index (API), respectively) and baPWV were measured. Estimated central BP (eCBP) was calculated from the data obtained from PASESA ${ }^{\circledR}$, and CBP was also measured simultaneously by invasive catheterization.
\end{abstract}

Results: API, but not AVI and baPWV, in the SVD group was significantly higher than that in the non-SVD group. Although eCBP was significantly associated with $\mathrm{CBP}$, there was no difference in eCBP between the groups. There were significant associations among API, AVI and baPWV, albeit these associations were relatively weak. A multivariate logistic regression revealed that API and $\beta$-blocker were significant independent variables that were associated with the presence of significant coronary stenosis. The cut-off level of API that gave the greatest sensitivity and specificity for the presence of SVD was 24 units (sensitivity 0.636 and specificity 0.667 ).

Conclusion: In conclusion, API, but not AVI or baPWV, is associated

Manuscript accepted for publication June 16, 2016

aDepartment of Cardiology, Fukuoka University School of Medicine, Fukuoka, Japan

${ }^{b}$ Department of Molecular Cardiovascular Therapeutics, Fukuoka University School of Medicine, Fukuoka, Japan

'Department of Rehabilitation, Fukuoka University Hospital, Fukuoka 8140180, Japan

${ }^{\mathrm{d} C o r r e s p o n d i n g ~ A u t h o r: ~ S h i n-i c h i r o ~ M i u r a, ~ D e p a r t m e n t ~ o f ~ C a r d i o l o g y, ~ F u k u o-~}$ ka University School of Medicine, 7-45-1 Nanakuma, Jonan-ku, Fukuoka 814-0180, Japan.Email: miuras@cis.fukuoka-u.ac.jp

doi: http://dx.doi.org/10.14740/jocmr2615w with the presence of significant coronary stenosis.

Keywords: Blood pressure; Arterial pulse velocity index; Arterial pulse amplitude index; Brachial-ankle pulse wave velocity; Stenosed coronary vessels

\section{Introduction}

Atrial stiffness is one of the major contributors to the detection of cardiovascular disease (CVD) [1,2], and the early detection of atrial stiffness can help to prevent CVD. We and others previously reported that non-invasive assessments, including pulse wave velocity (PWV) [3-6], carotid intima-media thickness [7, 8], flow-mediated vasodilatation [9] and augmentation index [10], could be used to detect atrial stiffness and the risk of CV. Although these assessments are accepted both clinically and experimentally, they take time and can present some technical difficulties. Thus, technologies simpler and easier to use are needed by physicians in the examination room. Komine et al recently developed a simple and non-invasive method for evaluating arterial stiffness using oscillometric measurements of blood pressure (BP) (PASESA ${ }^{\circledR}$ AVE-1500, Shisei Datum, Tokyo, Japan) [11]. This methodology is as easy to use as the physician simply measuring BP in the arm. A new index of arterial stiffness (arterial pressure volume index, API) has been shown to be related to brachial-ankle PWV (baPWV) $(\mathrm{r}=-0.53, \mathrm{P}<0.05)$, carotidfemoral PWV $(\mathrm{r}=-0.49, \mathrm{P}<0.05)$, and carotid arterial compliance $(\mathrm{r}=0.32, \mathrm{P}<0.05)$ [11]. Although these parameters are associated with each other, the associations are not strong.

More recently, with the use of parameters obtained by PASESA ${ }^{\circledR}$ (arterial velocity pulse index (AVI) and API), Sueta et al devised formulas to calculate estimated central systolic $\mathrm{BP}(\mathrm{eCSBP})$ and estimated central pulse pressure (eCPP) using intercepts and coefficients for independent variables [12, 13]. Although increased CBP causes vascular wall stress resulting in various CVDs $[14,15]$, the direct measurement of CBP is an invasive procedure that requires a catheter. Therefore, the noninvasive measurement of these parameters using PASESA ${ }^{\circledR}$ is important.

However, very little information is available on the association between various parameters obtained using PASESA ${ }^{\circledR}$ and the presence and severity of coronary artery disease (CAD). Consequently, we hypothesized that AVI and API could be bet- 
ter indicators of coronary atherosclerosis than conventional coronary risk factors in addition to PWV. In this study, we analyzed the associations between various parameters (AVI, API, eCSBP and eCPP) and the severity of CAD in patients with suspected CAD who underwent coronary angiography (CAG).

\section{Methods}

\section{Study population}

We enrolled 108 consecutive patients who underwent CAG due to suspected CAD in whom we could measure various parameters using PASESA ${ }^{\circledR}$ in addition to baPWV. The patients were divided into two groups: patients who did not have significantly stenosed coronary vessel disease $(n=33$, non-SVD group) and those who had at least one significantly stenosed coronary vessel ( $n=75$, SVD group). Our protocol was approved by the ethics committee of Fukuoka University Hospital. We retrospectively collected and analyzed all data using the database of Fukuoka University Hospital.

\section{Cardiovascular risk factors and biochemical parameters in blood}

We collected information regarding the patient's coronary risk factors including hypertension (HTN), diabetes mellitus (DM), dyslipidemia (DL) and smoking in addition to current medications. Height and weight were measured for all patients, and body mass index (BMI, weight $(\mathrm{kg}) /$ height $\left.(\mathrm{m})^{2}\right)$ was calculated. Data on biochemical parameters in blood including high-density lipoprotein cholesterol (HDL-C), low-density lipoprotein cholesterol (LDL-C), triglyceride (TG), glycated hemoglobin A1c (HbA1c), and estimated glomerular filtration rate (eGFR) were also collected.

\section{Measurements of AVI and API using PASESA ${ }^{\circledR}$}

We wrapped a cuff around the left upper arm of supine patients and simultaneously measured the brachial BP oscillometrically using PASESA ${ }^{\circledR}$ after at least 5 min of rest, as we measured CBP by heart catheterization. AVI, API, SBP, diastolic BP $(\mathrm{DBP})$, pulse rate $(\mathrm{PR})$ and $\mathrm{PP}$ were collected. We calculated eCSBP and eCPP as follows: eCSBP $=0.1152 \times$ age +0.7512 $\times \mathrm{SBP}+0.3095 \times \mathrm{DBP}+0.1884 \times \mathrm{AVI}+0.4001 \times \mathrm{API}-$ 0.1105 , and $\mathrm{eCPP}=0.1496 \times$ age $+0.1088 \times \mathrm{SBP}+0.7312 \times$ $\mathrm{PP}+0.2163 \times \mathrm{AVI}+0.3649 \times \mathrm{API}-12.3859[12,13]$.

\section{Measurements of baPWV and ankle-brachial index (ABI)}

Bilateral baPWV and ABI were measured during hospitalization. After the patient had rested in a supine position for at least $5 \mathrm{~min}$, baPWV was measured using a volume plethysmographic device (Omron Healthcare, Kyoto, Japan), which uses BP cuffs wrapped on the arm near the brachial artery and near the tibial artery of the ankle [16]. Mean baPWV (M. baPWV) and M. ABI were calculated as (lt. baPWV + rt. baPWV) $/ 2$ and (lt. $\mathrm{ABI}+$ rt. $\mathrm{ABI}) / 2$, respectively.

\section{Transthoracic ultrasound echocardiography (UCG)}

Echocardiography was performed upon hospitalization before CAG. An experienced sonographer obtained all echocardiographic data, which were interpreted by an experienced staff echocardiographer. Comprehensive examinations were performed in all of the study patients, including M-mode, twodimensional, conventional Doppler, and color Doppler echocardiography. The left ventricular ejection fraction (LVEF), LV dimension at end-diastole (LVDd), LV dimension at endsystole (LVDs), intraventricular septum (IVS), left ventricular posterior wall (LVPW) and E/A were collected.

\section{Heart catheterization}

A heart catheter was placed into the ascending aorta of patients, and CBP was measured and recorded prior to CAG. CAG was performed according to the Judkins technique by the patients' interventional cardiologists [17]. Coronary angiograms were analyzed with respect to the 15 -segment coding system of the American Heart Association [18], and significant SVD was considered to be $>50 \%$ diameter stenosis.

\section{Statistical analysis}

Statistical analysis was performed using the Stat View statistical software package (Stat View 5; SAS Institute Inc., Cary, NC, USA) at Fukuoka University (Fukuoka, Japan). Values are expressed as mean \pm standard deviation (SD). Categorical and continuous variables were compared between groups by a Chisquare analysis and Student's $t$-test, respectively. The Spearman Rank Correlation Coefficient was used to evaluate associations between the groups. A receiver-operating characteristic (ROC) curve analysis was used to determine the cut-off values of API to distinguish between non-SVD and SVD groups at the highest possible sensitivity and specificity. We also used a multiple logistic regression analysis to evaluate independent associated factors in the SVD group. Statistical significance was defined as a P-value less than 0.05 .

\section{Results}

\section{Patient characteristics in all patients and in the non-SVD and SVD groups}

The patient characteristics in all patients and in the non-SVD and SVD groups are shown in Table 1. Eight-six of the overall patients $(80 \%)$ were male. The mean ages of all patients and of the non-SVD and SVD groups were $70 \pm 10,68 \pm 9$ and $71 \pm$ 9 years, respectively. There were no significant differences in 
Table 1. Patient Characteristics in the All Patients, and in the Non-SVD and SVD Groups

\begin{tabular}{llll}
\hline & All $(\mathbf{n}=\mathbf{1 0 8})$ & Non-SVD $(\mathbf{n}=\mathbf{3 3})$ & SVD $(\mathbf{n}=\mathbf{7 5})$ \\
\hline Age, years & $70 \pm 10$ & $68 \pm 9$ & $71 \pm 9$ \\
Male, n (\%) & $86(80)$ & $24(76)$ & $61(81)$ \\
BMI, kg/m ${ }^{2}$ & $23.3 \pm 3.4$ & $23.2 \pm 4.0$ & $23.3 \pm 3.2$ \\
Smoking, n (\%) & $72(67)$ & $22(67)$ & $50(67)$ \\
HTN, n (\%) & $79(73)$ & $20(61)$ & $59(79)$ \\
DL, n (\%) & $77(71)$ & $17(52)$ & $60(80)^{* *}$ \\
HDL-C, mg/dL & $48 \pm 13$ & $51 \pm 11$ & $47 \pm 14$ \\
LDL-C, mg/dL & $99 \pm 33$ & $99 \pm 27$ & $98 \pm 36$ \\
TG, mg/dL & $134 \pm 79$ & $144 \pm 112$ & $130 \pm 60$ \\
DM, n (\%) & $47(43)$ & $9(27)$ & $38(50)^{*}$ \\
HbA1c, \% & $6.5 \pm 1.1$ & $6.4 \pm 1.1$ & $6.6 \pm 1.1$ \\
eGFR, mL/min/1.73 m ${ }^{2}$ & $60 \pm 19$ & $63 \pm 19$ & $59 \pm 18$ \\
Medication, n $(\%)$ & & & \\
\multicolumn{1}{c}{ ARB/ACE-I } & $68(64)$ & $18(55)$ & $50(68)$ \\
CCB & $60(56)$ & $17(52)$ & $43(58)$ \\
Nitrate & $15(14)$ & $1(3)$ & $14(19)^{*}$ \\
$\beta$ B-blocker & $46(46)$ & $9(27)$ & $37(50)^{*}$ \\
Statin & $77(72)$ & $18(55)$ & $59(80)^{*}$ \\
Insulin & $9(9)$ & $3(11)$ & $6(8)$ \\
OHA & $41(38)$ & $8(24)$ & $33(45)^{*}$ \\
\hline
\end{tabular}

BMI: body mass index; HTN: hypertension; DL: dyslipidemia; HDL-C: high-density lipoprotein cholesterol; LDL-C: low-density lipoprotein cholesterol; TG: triglyceride; DM: diabetes mellitus; HbA1c: hemoglobin A1C; eGFR: estimated glomerular filtration rate; ARB/ACE-I: angiotensin II receptor blocker/ angiotensin converting enzyme inhibitor; $\mathrm{CCB}$ : calcium channel blocker; $\mathrm{OHA}$ : oral hypoglycemic agent. ${ }^{*} P<0.05,{ }^{* *} P<0.01$ vs. non-SVD group.

patient characteristics between the non-SVD and SVD groups except for percentages (\%) of DL, DM and several kinds of medication. The SVD group showed significantly higher $\% \mathrm{DL}, \% \mathrm{DM}$, $\beta$-blocker, nitrate, statin and oral hypoglycemic agent (OHA) than the non-SVD group.

\section{Hemodynamic parameters in all patients and in the non- SVD and SVD groups}

There were no significant differences in hemodynamic parameters between the non-SVD and SVD groups except for API, as shown in Table 2 and Figure 1. While the SVD group showed significantly higher API than the non-SVD group $(\mathrm{P}=$ $0.03)$, there were no significant differences in $\mathrm{M}$. baPWV $(\mathrm{P}=$ $0.09)$ or AVI $(\mathrm{P}=0.13)$.

Figure 2 shows the association between CSBP and eCSBP and that between CPP and eCPP in all patients. eCSBP and eCPP were significantly and positively associated with CSBP $(\mathrm{r}=0.834, \mathrm{P}<0.0001)$ and $\mathrm{CPP}(\mathrm{r}=0.774, \mathrm{P}<0.0001)$, respectively.

Next, we analyzed the associations among AVI, API and baPWV (Fig. 3). baPWV was significantly and positively associated with AVI $(\mathrm{r}=0.373, \mathrm{P}<0.001)$ and API $(\mathrm{r}=0.280$,
$\mathrm{P}<0.01)$, and API was significantly and positively associated with AVI $(\mathrm{r}=0.428, \mathrm{P}<0.0001)$. Although all three parameters reflect the severity of arterial stiffness, the associations among them were relatively weak.

\section{Associated factors in the SVD group}

Finally, we analyzed the associated factors in the SVD group using independent variables (DL, DM, $\beta$-blocker, nitrate, statin and $\mathrm{OHA}$ in addition to age $\geq 65$ years, gender and $\mathrm{BMI})$ (Table 3). We selected DL, DM, $\beta$-blocker, nitrate, statin and $\mathrm{OHA}$ as independent variables because there were significant differences in these factors between the non-SVD and SVD groups, as shown in Table 1 . In a logistic regression analysis, AVI $(\mathrm{P}=0.042)$ and administration of $\beta$-blocker $(\mathrm{P}=0.033)$ were identified as significant independent variables that were associated with the SVD group.

\section{Cut-off value of API for predicting SVD}

The ROC curve analysis showed a higher area-under-the-curve for API (0.641) (Fig. 4). The cut-off level of API that gave the 
Table 2. Hemodynamic Parameters in the All Patients, and in the Non-SVD and SVD Groups

\begin{tabular}{|c|c|c|c|}
\hline & All $(n=108)$ & Non-SVD $(n=33)$ & $\operatorname{SVD}(n=75)$ \\
\hline \multicolumn{4}{|l|}{ PASESA $^{\circledR}$} \\
\hline $\mathrm{SBP}, \mathrm{mm} \mathrm{Hg}$ & $135 \pm 22$ & $130 \pm 21$ & $137 \pm 23$ \\
\hline DBP, mm Hg & $74 \pm 11$ & $75 \pm 9$ & $73 \pm 11$ \\
\hline $\mathrm{PP}, \mathrm{mm} \mathrm{Hg}$ & $61 \pm 20$ & $56 \pm 17$ & $64 \pm 20$ \\
\hline $\mathrm{PR}, \mathrm{mm} \mathrm{Hg}$ & $66 \pm 14$ & $69 \pm 16$ & $65 \pm 13$ \\
\hline AVI, unit & $32 \pm 10$ & $29 \pm 11$ & $33 \pm 10$ \\
\hline API, unit & $27 \pm 8$ & $24 \pm 8$ & $28 \pm 7 *$ \\
\hline eCSBP, mm Hg & $149 \pm 22$ & $144 \pm 21$ & $151 \pm 23$ \\
\hline eCPP, mm Hg & $74 \pm 21$ & $68 \pm 19$ & $77 \pm 21$ \\
\hline \multicolumn{4}{|l|}{ baPWV } \\
\hline Rt. baPWV, cm/s & $1,709 \pm 365$ & $1,618 \pm 326$ & $1,744 \pm 376$ \\
\hline Lt. baPWV, cm/s & $1,712 \pm 354$ & $1,620 \pm 285$ & $1,749 \pm 374$ \\
\hline M. baPWV, $\mathrm{cm} / \mathrm{s}$ & $1,710 \pm 355$ & $1,619 \pm 303$ & $1,747 \pm 369$ \\
\hline \multicolumn{4}{|l|}{ ABI } \\
\hline Rt. ABI & $1.11 \pm 0.12$ & $1.10 \pm 0.14$ & $1.11 \pm 0.12$ \\
\hline Lt. ABI & $1.10 \pm 0.10$ & $1.09 \pm 0.10$ & $1.10 \pm 0.10$ \\
\hline M. ABI & $1.10 \pm 0.10$ & $1.10 \pm 0.11$ & $1.11 \pm 0.10$ \\
\hline \multicolumn{4}{|l|}{ UCG } \\
\hline LVEF, \% & $59 \pm 13$ & $60 \pm 15$ & $59 \pm 13$ \\
\hline LVDd, mm & $46 \pm 8$ & $45 \pm 7$ & $46 \pm 8$ \\
\hline LVDs, mm & $31 \pm 8$ & $31 \pm 8$ & $32 \pm 8$ \\
\hline IVS, mm & $9.8 \pm 1.8$ & $9.5 \pm 2.2$ & $10.0 \pm 1.6$ \\
\hline LVPW, mm & $10.3 \pm 1.7$ & $9.9 \pm 1.5$ & $10.4 \pm 1.7$ \\
\hline $\mathrm{E} / \mathrm{A}$ & $0.9 \pm 0.5$ & $1.0 \pm 0.8$ & $0.8 \pm 0.4$ \\
\hline \multicolumn{4}{|l|}{ Heart catheterization } \\
\hline CSBP, mm Hg & $149 \pm 30$ & $144 \pm 34$ & $151 \pm 28$ \\
\hline CDBP, mm Hg & $66 \pm 13$ & $96 \pm 17$ & $95 \pm 17$ \\
\hline CPP, mm Hg & $82 \pm 27$ & $75 \pm 30$ & $85 \pm 26$ \\
\hline
\end{tabular}

SBP: systolic blood pressure; DBP: diastolic BP; PP: pulse pressure; PR: pulse rate; AVI: arterial velocity pulse index; API: arterial pressure volume index; eCSBP: estimated central SBP: baPWV: brachialankle pulse wave velocity; Rt: right; Lt: left; M: mean; ABI: ankle-brachial pressure index; UCG: ultrasound echocardiography; LVEF: left ventricular ejection fraction; LVDd: LV dimension at end-diastole; LVDs: LV dimension at end-systole; IVS: intraventricular septum; LVPW: left ventricular posterior wall. ${ }^{*} \mathrm{P}<0.05$ vs. non-SVD group.

greatest sensitivity and specificity for the presence of SVD was 24 units (sensitivity 0.636 and specificity 0.667 ).

\section{Discussion}

The major findings of the present study are as follows: API, but not AVI or baPWV, in the SVD group was significantly higher than that in the non-SVD group, and a multivariate logistic regression analysis revealed that API and administration of $\beta$-blocker were significant independent variables that were associated with the presence of significant coronary stenosis. In addition, while API, AVI and baPWV were significantly associated with each other, these associations were relatively weak. There was no difference in eCBP between the non-SVD and SVD groups, although eCBP was significantly associated with CBP.

The most interesting observation in this study was that API and administration of $\beta$-blocker were identified as significant independent variables that were associated with the presence of significant coronary stenosis. API can reflect brachial arterial stiffness [11]. Little is known about the significance of API in a clinical setting. One report showed that API was associated with eCBP and brachial PP in patients undergoing hemodialysis [19]. On the other hand, many previous reports have indicated that baPWV is an important parameter in the 

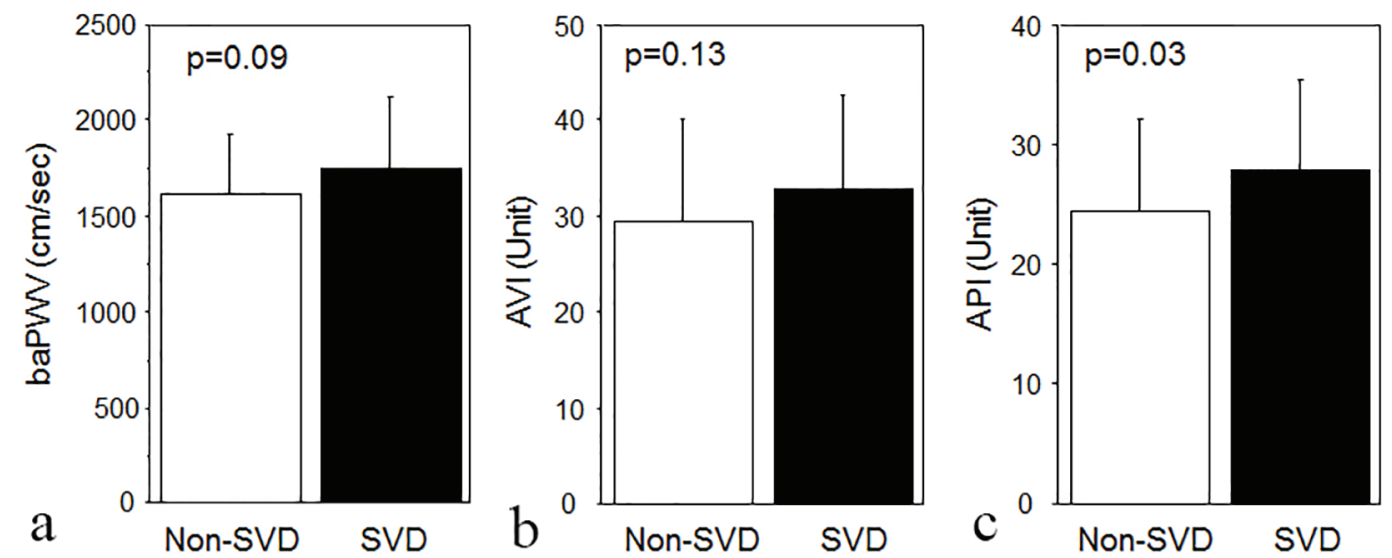

Figure 1. Brachial-ankle pulse wave velocity (baPWV) (a), arterial velocity pulse index (AVI) (b), and arterial pressure volume index (API) (c) in patients who did not have significant stenosed coronary vessel disease (non-SVD group, open bars) and in those who had at least one significantly stenosed coronary vessel (SVD group, closed bars).
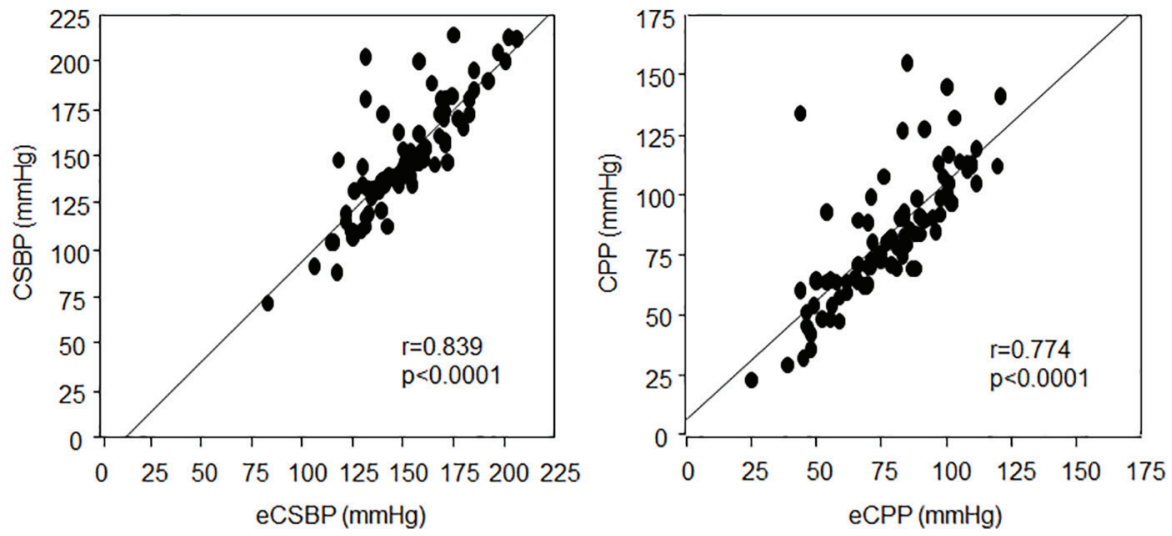

Figure 2. Associations between central systolic blood pressure (CSBP) and estimated CSBP (eCSBP) and between central pulse pressure (CPP) and estimated CPP (eCPP) in all patients.

progression of atherosclerotic CVD. Increased baPWV is associated with the development of endothelial dysfunction [20, 21], and higher levels of baPWV have been associated with the risk and severity of CAD [22]. Endothelial dysfunction led to stent revascularization in patients with CAD [23]. baPWV was a significant predictor of $\mathrm{CV}$ events in patients with chronic
CAD [20], and baPWV can be used as a risk-stratification index for the short-term prognosis in clinical practice [6]. In this study, baPWV in the SVD group tended to be higher than that in the non-SVD group, but this difference was not significant. Although arterial stiffness as assessed by baPWV is considered to be clinically significant, API, but not baPWV, was sig-
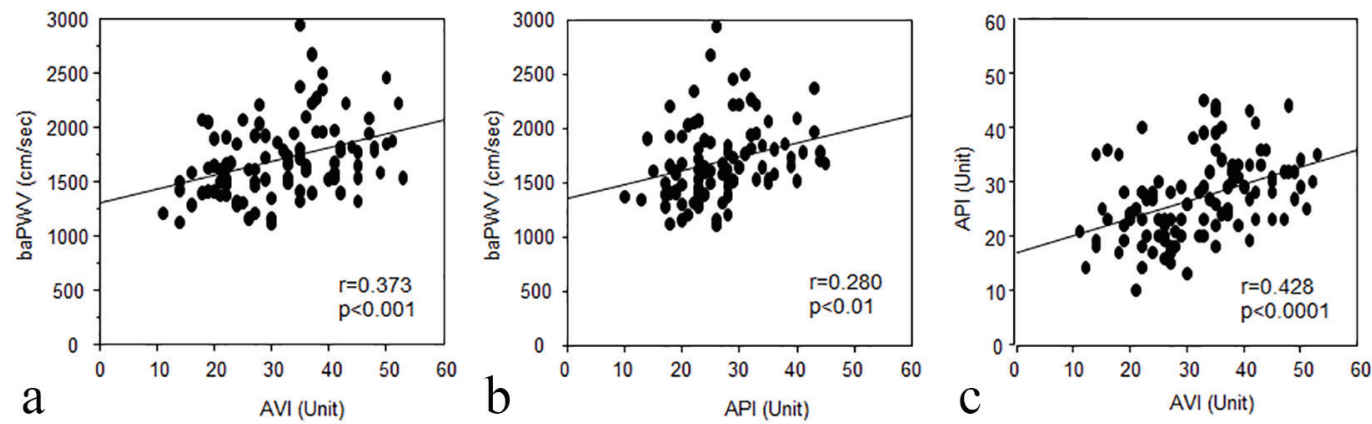

Figure 3. Associations between brachial-ankle pulse wave velocity (baPWV) and arterial velocity pulse index (AVI) (a), baPWV and arterial pressure volume index (API) (b), and AVI and API (c). 


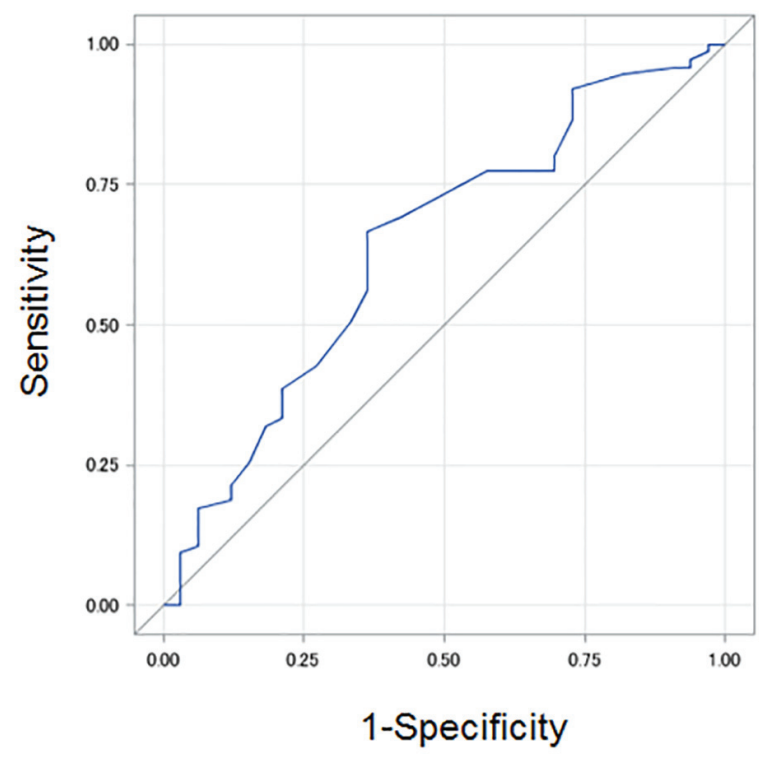

Figure 4. Cut-off value of arterial pressure volume index (API) in patients with at least one significantly stenosed coronary vessel (SVD group) by a receiver-operating characteristic (ROC) curve analysis.

nificantly associated with the presence of significant coronary stenosis. Thus, API might be a better tool than baPWV in the presence of stenosis. In addition, API and baPWV were significantly associated with each other, albeit this association was relatively weak. Although both parameters are indicators of arterial stiffness, API and baPWV reflect brachial (peripheral) arterial stiffness [11] and central stiffness in larger arteries, respectively. Thus, these assessments of stiffness do not evaluate the same artery. This difference might affect the association between API and baPWV. Further studies will be needed to resolve these issues.

The administration of $\beta$-blocker was also identified as a significant independent variable that was associated with the presence of significant coronary stenosis. This finding is reasonable because $\beta$-blocker is the first choice of medication in patients with effort angina [24], and because \%HTN in the SVD group was higher than that in the non-SVD group, although this difference was not statistically significant. Age is known to be associated with atrial stiffness, and age was associated with API $(\mathrm{r}=0.340, \mathrm{P}<0.001)$ and baPWV $(\mathrm{r}=0.553$, $\mathrm{P}<0.0001)$ in this study. Various parameters obtained using PASESA ${ }^{\circledR}$ including API could be influenced by various medications. To resolve these issues, we analyzed the factors associated with SVC using independent variables including age, $\beta$-blocker, nitrate, statin and OHA by a multivariate logistic regression, as shown in Table 3.

Although eCBP and eCPP were significantly associated with $\mathrm{CBP}$ and $\mathrm{CPP}$, respectively, there were no differences in eCBP and eCPP between the non-SVD and SVD groups. Previous reports have indicated that increased CBP caused vascular wall stress resulting in CVD $[14,15]$. Although AVI can reflect central arterial stiffness $[12,13]$ and was significantly associated with CSBP $(\mathrm{r}=0.543, \mathrm{P}<0.0001)$, there was also no difference in AVI between the groups. Thus, central arterial stiffness is not an important factor for the presence of coronary stenosis in this study. Instead, the non-invasive measurement of CBP using PASESA ${ }^{\circledR}$ is important, since direct CBP measurements are invasive. We used PASESA ${ }^{\circledR}$ to confirm the accuracy of eCSBP and eCPP calculated using formulas [12, 13], since eCSBP and eCPP were strongly associated with CSBP and CPP, respectively.

The present study has several limitations. First, this study was performed in a single center and the number of patients was relatively small. Second, the present study did not include healthy volunteers. Third, we did not take into account whether the patients had previously undergone coronary intervention. Nonetheless, API was significantly associated with the data obtained by CAG regarding the present conditions of the coronary arteries. Despite these limitations, this is the first demonstration of the association between a new vascular index and coronary stenosis, and suggests that the measurement of API may be clinically useful for screening significant coronary stenosis.

In conclusion, API, but not AVI or baPWV, is associated with the presence of significant coronary stenosis. The cut-off level of API for the presence of stenosis was 24 units.

\section{Conflict(s) of Interest/Disclosure(s)}

KS is a Chief Director and SM is a Director of NPO Clinical and Applied Science, Fukuoka, Japan. KS has an Endowed Department of "Department of Molecular Cardiovascular Therapeutics" supported by MSD, Co. Ltd. SM belongs to the Department of Molecular Cardiovascular Therapeutics supported by MSD, Co. Ltd.

\section{References}

1. Weber T, Auer J, O'Rourke MF, Kvas E, Lassnig E, Berent $\mathrm{R}$, Eber B. Arterial stiffness, wave reflections, and the risk of coronary artery disease. Circulation. 2004;109(2):184189.

2. Najjar SS, Scuteri A, Lakatta EG. Arterial aging: is it an immutable cardiovascular risk factor? Hypertension. 2005;46(3):454-462.

3. Laurent S, Boutouyrie P, Asmar R, Gautier I, Laloux B, Guize L, Ducimetiere P, et al. Aortic stiffness is an independent predictor of all-cause and cardiovascular mortality in hypertensive patients. Hypertension. 2001;37(5):1236-1241.

4. Tomiyama H, Koji Y, Yambe M, Shiina K, Motobe K, Yamada J, Shido N, et al. Brachial - ankle pulse wave velocity is a simple and independent predictor of prognosis in patients with acute coronary syndrome. Circ J. 2005;69(7):815-822.

5. Mitsutake R, Miura S, Saku K. Association between coronary artery calcification score as assessed by multi-detector row computed tomography and upstroke time of pulse wave. Intern Med. 2007;46(22):1833-1836.

6. Nakamura M, Yamashita T, Yajima J, Oikawa Y, Sagara K, Koike A, Kirigaya H, et al. Brachial-ankle pulse wave 
velocity as a risk stratification index for the short-term prognosis of type 2 diabetic patients with coronary artery disease. Hypertens Res. 2010;33(10):1018-1024.

7. Davis PH, Dawson JD, Riley WA, Lauer RM. Carotid intimal-medial thickness is related to cardiovascular risk factors measured from childhood through middle age: The Muscatine Study. Circulation. 2001;104(23):28152819.

8. Salonen JT, Salonen R. Ultrasonographically assessed carotid morphology and the risk of coronary heart disease. Arterioscler Thromb. 1991;11(5):1245-1249.

9. Koyoshi R, Miura S, Kumagai N, Shiga Y, Mitsutake R, Saku K. Clinical significance of flow-mediated dilation, brachial intima-media thickness and pulse wave velocity in patients with and without coronary artery disease. Circ J. 2012;76(6):1469-1475.

10. Goto T, Ohte N, Fukuta H, Wakami K, Tani T, Kimura G. Relationship between effective arterial elastance, total vascular resistance, and augmentation index at the ascending aorta and left ventricular diastolic function in older women. Circ J. 2013;77(1):123-129.

11. Komine H, Asai Y, Yokoi T, Yoshizawa M. Non-invasive assessment of arterial stiffness using oscillometric blood pressure measurement. Biomed Eng Online. 2012;11:6.

12. Sueta D, Kaikita K, Okamoto N, Arima Y, Ishii M, Ito M, Oimatsu Y, et al. A novel quantitative assessment of whole blood thrombogenicity in patients treated with a non-vitamin K oral anticoagulant. Int J Cardiol. 2015;197:98-100.

13. Sueta D, Yamamoto E, Tanaka T, Hirata Y, Sakamoto K, Tsujita K, Kojima S, Nishiyama K, Kaikita K, Hokimoto S, Jinnouchi H, Ogawa H. Association of estimated central blood pressure measured non-invasively with pulse wave velocity in patients with coronary artery disease. IJC Heart Vesc. 2015;8:52-54.

14. Williams B, Lacy PS, Thom SM, Cruickshank K, Stanton A, Collier D, Hughes AD, et al. Differential impact of blood pressure-lowering drugs on central aortic pressure and clinical outcomes: principal results of the Conduit Artery Function Evaluation (CAFE) study. Circulation. 2006;113(9):1213-1225.

15. Roman MJ, Devereux RB, Kizer JR, Lee ET, Galloway JM, Ali T, Umans JG, et al. Central pressure more strongly relates to vascular disease and outcome than does brachial pressure: the Strong Heart Study. Hypertension.
2007;50(1):197-203.

16. Asmar R, Benetos A, Topouchian J, Laurent P, Pannier B, Brisac AM, Target R, et al. Assessment of arterial distensibility by automatic pulse wave velocity measurement. Validation and clinical application studies. Hypertension. 1995;26(3):485-490.

17. Shah A, Gnoj J, Fisher VJ. Complications of selective coronary arteriography by the Judkins technique and their prevention. Am Heart J. 1975;90(3):353-359.

18. Austen WG, Edwards JE, Frye RL, Gensini GG, Gott VL, Griffith LS, McGoon DC, et al. A reporting system on patients evaluated for coronary artery disease. Report of the Ad Hoc Committee for Grading of Coronary Artery Disease, Council on Cardiovascular Surgery, American Heart Association. Circulation. 1975;51(4 Suppl):5-40.

19. Sueta D, Yamamoto E, Hirata Y, Tokitsu T, Sakamoto K, Tsujita K, Kaikita K, Hokimoto S, Sakanashi T, Ogawa H. Novel Vascular Indices Evaluated Non-Invasively in End-stage Renal Disease Patients on Hemodialysis. Clinical Trials and Regulatory Science in Cardiology. 2015;9:1-3

20. Sugamata W, Nakamura T, Uematsu M, Kitta Y, Fujioka D, Saito Y, Kawabata K, et al. Combined assessment of flow-mediated dilation of the brachial artery and brachial-ankle pulse wave velocity improves the prediction of future coronary events in patients with chronic coronary artery disease. J Cardiol. 2014;64(3):179-184.

21. Liu DH, Wang Y, Liao XX, Xu MG, Wang JM, Yang Z, Chen $\mathrm{L}$, et al. Increased brachial-ankle pulse wave velocity is associated with impaired endothelial function in patients with coronary artery disease. Chin Med J (Engl). 2006;119(22):1866-1870.

22. Xiong Z, Zhu C, Zheng Z, Wang M, Wu Z, Chen L, Chen Y. Relationship between arterial stiffness assessed by brachial-ankle pulse wave velocity and coronary artery disease severity assessed by the SYNTAX score. J Atheroscler Thromb. 2012;19(11):970-976.

23. Munk PS, Butt N, Larsen AI. Endothelial dysfunction predicts clinical restenosis after percutaneous coronary intervention. Scand Cardiovasc J. 2011;45(3):139-145.

24. Shimamoto K, Ando K, Fujita T, Hasebe N, Higaki J, Horiuchi M, Imai Y, et al. The Japanese Society of Hypertension Guidelines for the Management of Hypertension (JSH 2014). Hypertens Res. 2014;37(4):253-390. 